\title{
Blood pressure variability and obstructive sleep apnea. A question of phenotype?
}

\author{
Grzegorz Bilo ${ }^{1,2} \cdot$ Martino F. Pengo $^{1} \cdot$ Carolina Lombardi $^{1,2} \cdot$ Gianfranco Parati $^{1,2}$
}

Received: 9 September 2018 / Revised: 9 October 2018 / Accepted: 9 October 2018 / Published online: 5 November 2018

(c) The Japanese Society of Hypertension 2018

Obstructive sleep apnea (OSA) is a condition extremely prevalent among hypertensive patients and evidence is also available indicating its relationship with incident hypertension. Nonetheless, whether OSA actually causes hypertension is a complex question, because both these conditions share several predisposing factors, the most important being obesity [1]. Moreover, the character of the relationship between OSA and blood pressure (BP) depends on the range of time wherein BP is assessed. On one hand there is little doubt that apneas cause acute nocturnal BP surges due to a combination of chemoreflex-mediated sympathetic activation and changes in ventricular filling caused by dramatic variations of intrathoracic pressure during obstructive events. In fact, particularly in patients with severe OSA in whom apneas may occur once a minute or even more frequently, an increase in average nocturnal BP level can be reasonably expected. Indeed, as observed by means of 24-h ambulatory blood pressure monitoring, patients with severe OSA tend to present blunted nocturnal fall of BP (so called non-dipping profile) [2]. On the other hand, patients with severe OSA frequently have a constantly increased basal sympathetic tone, which might at least in part explain the occurrence of elevated $\mathrm{BP}$ also during the day $[1,3]$.

The intermittent occurrence of a phenomenon with a major direct impact on BP such as obstructive apneas inevitably raises questions on the possible relationship between OSA and $\mathrm{BP}$ variations over time, i.e. $\mathrm{BP}$ variability (BPV). BPV is a multifaceted phenomenon with numerous determinants, which may be described by a number of different estimates and over different periods of time, ranging from seconds to

Grzegorz Bilo

g.bilo@auxologico.it

1 Istituto Auxologico Italiano, IRCCS, Cardiology Unit, Ospedale San Luca, Milan, Italy

2 Department of Medicine and Surgery, University of Milano-Bicocca, Milan, Italy years [4]. For a long time research on BPV had focused mainly on short-term BP variations, occurring within 24-h. A few years ago, however, Rothwell et al. published a study indicating that also long-term BPV, assessed on a visit-tovisit basis (visit-to-visit variability; VVV) is able to predict cardiovascular outcomes [5]. Although the understanding of the underlying mechanisms and pathophysiological consequences of VVV remains incomplete, its association with adverse outcomes was confirmed in a number of subsequent studies [6].

Consistent evidence indicates that the presence of OSA may be associated with increased short-term BPV but until now the information on its relationship with VVV was limited. Shiina et al. observed that patients with severe OSA $(n=35)$ had significantly higher systolic VVV than controls $(n=26)$ matched for age, BMI and systolic BP [7]. Moreover, in this study apnea-hypopnea index (AHI, the principal index of OSA severity) emerged as a significant predictor of VVV in multivariable analysis and VVV was significantly reduced in subjects treated with continuous positive airway pressure (CPAP) and adherent to this treatment. In a different study Pengo et al. also found a significant reduction in systolic BPV after 2 weeks of autotitrating CPAP treatment in OSA patients, but in this case BPV was estimated as standard deviations (SD) of values obtained during the same visit (within-visit variability) rather than on a visit-to-visit basis [8].

On this background the paper by Kansui et al. published in the current issue of Hypertension Research provides further interesting evidence on the association between OSA and VVV [9]. The study included employees of a transport company in Japan, who were systematically screened for the presence of OSA by means of nocturnal pulse oximetry, followed by polysomnography whenever the initial screening produced pathological results (4\% oxygen desaturation index [ODI] $\geq 10 / \mathrm{h}$ ). Out of 1653 initially screened participants 131 were found to have mildto-moderate OSA $(5 \leq \mathrm{AHI}<30)$ and 108 had severe OSA (AHI $\geq 30$ ). BPV was subsequently assessed by calculating SDs and coefficients of variation $(\mathrm{CV})$ of $\mathrm{BP}$ values 
obtained during annual health checkups over 4 years' follow-up. The authors found that, after adjusting for major covariates, systolic VVV was significantly increased in subjects with severe OSA compared with mild-to-moderate OSA and healthy controls. Conversely, treatment with CPAP was associated with lower systolic VVV [9].

Even though the study by Kansui et al. seems to mostly confirm the data of Shiina et al. there are several relevant differences between the two studies. First, the former study included a significantly larger number of participants derived from a general worksite sample, whereas the latter was performed in a sample of patients preselected based on clinical referral criteria. Although the non-OSA controls in the former sample were not matched and thus had significantly different characteristics compared with OSA patients (in particular the latter had higher BMI, BP level, rate of hypertension prevalence and presence of antihypertensive treatment), the sample size allowed to adjust for these confounders in multivariable analysis. Second, the intervals between BP measurements differed significantly between the two studies: in the study by Kansui et al. BP was assessed yearly, while Shiina et al. performed monthly evaluations. Such a difference may be relevant as monthly evaluations could be affected by e.g. seasonal changes of BP, while yearly assessments are more similar to what is normally done in clinical practice [7, 9].

Some other aspects of the study by Kansui et al. are of interest. The association between OSA and VVV was evident in multivariable analysis, whereas unadjusted data produced similar mean values of VVV estimates in severe OSA as in non-OSA participants, and even lower crude values were reported in those with mild-to-moderate OSA [9]. Such findings imply the presence of negative confounders. One of them is surely the effect of OSA treatment with CPAP or oral appliances, which was significantly associated with lower VVV both in the entire sample and in the subgroup of participants who proceeded to polysomnographic evaluation. The mechanism underlying the association between OSA treatment and lower VVV is not clear: it might represent a true effect of the treatment, acting on the pathophysiologic mechanisms responsible for BP variations in OSA, but it may also reflect a generally more accurate care of subjects receiving this specific form of treatment.

Also the possible mechanisms linking OSA with VVV are unclear. Changes in antihypertensive treatment during followup may contribute to elevated VVV (treatment titration may increase BPV due to progressive BP lowering during consecutive visits). In fact, when the authors limited the analysis to participants in whom no changes in antihypertensive treatment were made, the differences in VVV between groups were attenuated, although the tendency to a higher VVV in subjects with severe OSA persisted. As far as pathophysiological mechanisms are concerned, it is not easy to indicate one which might directly link the presence of OSA and increased VVV. It seems reasonable to think, though, that this association is not a manifestation of a cause-effect relationship. Instead, both these features may represent different facets of a complex high-risk phenotype combining obesity, presence of sleep apnea, sympathetic overactivity, and impaired cardiovascular homeostatic control, reflected by increased VVV. Considering that the observed OSA-related differences regarded systolic and not diastolic BPV, also the involvement of increased arterial stiffness cannot be excluded, even if in the previous study of Shiina et al. brachial-ankle pulse wave velocity did not differ between OSA and nonOSA participants [7]. Finally, other factors, such as sleep quality and quantity and the presence of excessive daytime sleepiness before and after treatment could be relevant but were not considered in the analyses performed by Kansui et al. [9].

Considering the general complexity of BPV phenomena, further research is clearly required to fully understand how the presence of OSA may be related with different BPV estimates and whether this relationship may have clinical relevance.

\section{Compliance with ethical standards}

Conflict of interest The authors declare that they have no conflict of interest.

\section{References}

1. Parati G, Ochoa JE, Bilo G, Mattaliano P, Salvi P, Kario K, et al. Obstructive sleep apnea syndrome as a cause of resistant hypertension. Hypertens Res. 2014;37:601-13.

2. Wolf J, Hering D, Narkiewicz K. Non-dipping pattern of hypertension and obstructive sleep apnea syndrome. Hypertens Res. 2010;33:867-71.

3. Bisogni V, Pengo MF, Maiolino G, Rossi GP. The sympathetic nervous system and catecholamines metabolism in obstructive sleep apnoea. J Thorac Dis. 2016;8:243-54.

4. Parati G, Ochoa JE, Lombardi C, Bilo G. Assessment and management of blood-pressure variability. Nat Rev Cardiol. 2013;10:143-55.

5. Rothwell PM, Howard SC, Dolan E, O'Brien E, Dobson JE, Dahlöf $\mathrm{B}$, et al. Prognostic significance of visit-to-visit variability, maximum systolic blood pressure, and episodic hypertension. Lancet. 2010;375:895-905.

6. Stevens SL, Wood S, Koshiaris C, Law K, Glasziou P, Stevens RJ, et al. Blood pressure variability and cardiovascular disease: systematic review and meta-analysis. BMJ. 2016;354:i4098.

7. Shiina K, Tomiyama H, Takata Y, Matsumoto C, Odaira M, Kato $\mathrm{K}$, et al. Obstructive sleep apnea as possible causal factor for visitto-visit blood pressure variability. Circ J. 2016;80:1787-94.

8. Pengo MF, Ratneswaran C, Berry M, Kent BD, Kohler M, Rossi $\mathrm{GP}$, et al. Effect of continuous positive airway pressure on blood pressure variability in patients with obstructive sleep apnea. J Clin Hypertens. 2016;18:1180-4.

9. Kansui Y, Matsumura K, Inoue M, Sakata S, Oishi E, Goto K, et al. Impact of obstructive sleep apnea on long-term blood pressure variability in Japanese men: a cross-sectional study of a work-site population. Hypertens Res. 2018;0:00-00. 